\title{
Effect of peritoneal dialysis fluid containing osmo- metabolic agents on human endothelial cells
}

\author{
This article was published in the following Dove Press journal: \\ Drug Design, Development and Therapy \\ 28 November 2016 \\ Number of times this article has been viewed
}

\author{
Mario Bonomini ${ }^{1,2}$ \\ Sara Di Silvestre ${ }^{3,4}$ \\ Pamela Di Tomo ${ }^{3,4}$ \\ Natalia Di Pietro 2,4 \\ Domitilla Mandatori ${ }^{3,4}$ \\ Lorenzo Di Liberato' \\ Vittorio Sirolli ${ }^{1,2}$ \\ Francesco Chiarelli ${ }^{2,4}$ \\ Cesare Indiveri $\mathrm{i}^{5}$ \\ Assunta Pandolfi $\mathrm{f}^{3,4}$ \\ Arduino Arduini ${ }^{6}$ \\ 'Unit of Nephrology and Dialysis, \\ ${ }^{2}$ Department of Medicine and \\ Aging Sciences, ${ }^{3}$ Department of \\ Medical, Oral and Biotechnological \\ Sciences, ${ }^{4}$ Aging Research Center \\ and Translational Medicine, CeSI-MeT, \\ University “G. d'Annunzio", \\ Chieti-Pescara, ${ }^{5}$ Department DiBEST \\ (Biologia, Ecologia, Scienze della \\ Terra), Unit of Biochemistry and \\ Molecular Biotechnology, University \\ of Calabria, Arcavacata di Rende CS, \\ Italy; ${ }^{6}$ CoreQuest, Manno, Switzerland
}

Correspondence: Mario Bonomini

Unit of Nephrology and Dialysis,

SS. Annunziata Hospital, Via dei Vestini,

660I 3 Chieti, Italy

Tel +39 087I 540 I20

Fax +39 087I 574736

Email m.bonomini@nephro.unich.it
Background: The use of glucose as the only osmotic agent in peritoneal dialysis (PD) solutions (PDSs) is believed to exert local (peritoneal) and systemic detrimental actions, particularly in diabetic PD patients. To improve peritoneal biocompatibility, we have developed more biocompatible PDSs containing xylitol and carnitine along with significantly less amounts of glucose and have tested them in cultured Human Vein Endothelial Cells (HUVECs) obtained from the umbilical cords of healthy (C) and gestational diabetic (GD) mothers.

Methods: Primary C- and GD-HUVECs were treated for 72 hours with our PDSs (xylitol $0.7 \%$ and $1.5 \%$, whereas carnitine and glucose were fixed at $0.02 \%$ and $0.5 \%$, respectively) and two glucose-based PDSs (glucose $1.36 \%$ or $2.27 \%$ ). We examined their effects on endothelial cell proliferation (cell count), viability (3-(4,5-dimethylthiazolyl-2)-2,5-diphenyltetrazolium bromide assay), intracellular nitro-oxidative stress (peroxynitrite levels), Vascular Cell Adhesion Molecule-1 and Intercellular Adhesion Molecule-1 membrane exposure (flow cytometry), and HUVEC-monocyte interactions (U937 adhesion assay).

Results: Compared to glucose-based PDSs, our in vitro studies demonstrated that the tested PDSs did not change the proliferative potential both in C- and GD-HUVECs. Moreover, our PDSs significantly improved endothelial cell viability, compared to glucose-based PDSs and basal condition. Notably, glucose-based PDSs significantly increased the intracellular peroxynitrite levels, Vascular Cell Adhesion Molecule-1 and Intercellular Adhesion Molecule-1 membrane exposure, and endothelial cell-monocyte interactions in both C- and GD-HUVECs, as compared with our experimental PDSs.

Conclusion: Present results show that in control and diabetic human endothelial cell models, xylitol-carnitine-based PDSs do not cause cytotoxicity, nitro-oxidative stress, and inflammation as caused by hypertonic glucose-based PDSs. Since xylitol and carnitine are also known to favorably affect glucose homeostasis, these findings suggest that our PDSs may represent a desirable hypertonic solution even for diabetic patients in PD.

Keywords: carnitine, peritoneal dialysis solution, inflammation, nitro-oxidative stress, endothelial cells, xylitol

\section{Introduction}

Peritoneal dialysis (PD) is a well-established mode of renal replacement therapy for patients suffering from end-stage renal disease, and has been used by approximately $11 \%$ of dialysis patients worldwide. ${ }^{1}$ It is primarily a home-based treatment, which can be performed manually (continuous ambulatory PD [CAPD]) or employing a mechanical device (automated PD).

PD is based on the exchange of solutes and fluid between the peritoneal capillary blood and a solution (dialysate) introduced into the peritoneal cavity through an implanted catheter. PD solution (PDS) contains electrolytes, a buffer (lactate or 
bicarbonate), and an osmotic agent needed to remove excess water from the patient's body (peritoneal ultrafiltration). Glucose is the osmotic agent almost universally used in PD due to its acceptable safety profile, efficacy, delivery of energy source, and low cost. Regulatory wise, the active osmotic ingredient present in PDSs is regarded as a drug and it must go through the traditional drug development process in order to achieve market authorization by the US Food and Drug Administration and by the European Medicines Agency.

PD therapy may provide a clinical outcome comparable to hemodialysis (HD) $)^{2}$ and an even better quality of life. ${ }^{3,4}$ In addition, several studies underpin the important link between maintained residual renal function (RRF) in PD and survival benefit, ${ }^{5-7}$ whereas HD is linked with a loss of RRF compared with that observed in PD. ${ }^{8}$

However, a major Achilles' heel of the treatment is still represented by the poor local and systemic biocompatibility of current standard PDSs. Though several factors have been alleged, ${ }^{9}$ glucose along with toxic glucose degradation products (GDPs) generated during heat sterilization of glucose-based solutions ${ }^{10}$ is by far thought as the main culprit for the bioincompatibility of PDS. ${ }^{11}$ Glucose has been associated with functional and morphological damage to the peritoneal membrane ${ }^{12}$ and to vascular cells such as the endothelial cells. ${ }^{13,14}$ Glucose and GDPs also induce apoptosis of peritoneal mesothelial cells and endothelial cells and, in particular, GDPs show a stronger reactivity than glucose in the formation of advanced glycation end-products, a known cause for microvascular complications and arteriosclerosis. ${ }^{15}$ Moreover, excessive glucose absorption (up to $200 \mathrm{~g} /$ day) may cause or aggravate metabolic disturbances frequently encountered in end-stage renal disease, such as dyslipidemia, insulin resistance, hyperinsulinemia, inflammation, and altered adipokine levels. ${ }^{16}$ Although PD has been traditionally considered a more physiological technique than HD, these results raise some doubts with respect to inflammation and endothelial damage. ${ }^{17}$

Based on these evidences, is not surprising that strategies designed to reduce/eliminate glucose-associated toxicity form one of the modern goals of PD. ${ }^{11,18}$

A novel glucose-sparing approach may be represented by the use of osmo-metabolic agents in the PDS that are not only able to reduce intraperitoneal glucose load without compromising ultrafiltration, but also to independently mitigate underlying metabolic disorders. ${ }^{19}$ Osmo-metabolic agents may be used singly, or in combination in order to maximize their therapeutic effects. In this context, our recent studies support the use of L-carnitine, which is involved in the mitochondrial oxidation of long-chain fatty acids, ${ }^{20}$ as a suitable osmotic agent in PD. ${ }^{21}$ The presence of L-carnitine in the solution was safe and well tolerated, ${ }^{21,22}$ and proved to be more biocompatible than glucose in several experimental models. ${ }^{21,23}$ In addition, a PDS containing L-carnitine significantly increased insulin sensitivity in a 4-month randomized controlled study in nondiabetic CAPD patients. ${ }^{22}$

Furthermore, a study conducted some years ago highlights the potential beneficial effect of xylitol, ${ }^{24}$ which is involved in the pentose phosphate shunt and has low glycemic properties. ${ }^{25}$ Effect of xylitol used for at least 5 months as an osmotic agent fully replacing glucose in the PD fluid of six type 1 diabetic patients on CAPD proved to be safe, maintained peritoneal ultrafiltration, and significantly improved the glycemic control. ${ }^{24}$

Aside from the different types of agents that may replace glucose, when developing a new PDS, one should also examine its impact on the peritoneal membrane, which consists of three layers: the capillary endothelium, the interstitium, and the mesothelium. ${ }^{26}$ In PD therapy, the capillary endothelium is the major barrier of the peritoneum to the transport of water and solutes. In addition, it progressively emerged that the microvascular endothelium is not only a permeability barrier and a thromboresistant surface, but also the location of relevant synthetic and metabolic activities. $^{27}$

In the present study, we investigated the biocompatibility of a new experimental PDS containing L-carnitine, xylitol, and low amount of glucose, instead of glucose alone, on Human Vein Endothelial Cells (HUVECs) obtained from umbilical cords of healthy mothers and of mothers suffering from gestational diabetes (GD). GD is associated with increased oxidative stress, inflammation, and overexpression of inflammatory cytokines, ${ }^{14,28}$ which are the common abnormalities in patients on PD. ${ }^{29-31}$

\section{Methods}

\section{Antibodies and materials}

Experimental PDSs (Table 1) were formulated in order to achieve an osmolarity (calculated) comparable to that of the commercially available low-GDPs glucose-based PDSs (Physioneal 40, glucose $1.36 \%$ or $2.27 \%$; Baxter Healthcare, Mc Gaw Park, IL, USA), steam-sterilized, and provided in sterile disposable 2 L bags (HBiofluids Srl, Tovo S. Agata, Italy). In particular, xylitol and carnitine concentrations were selected according to the current approved dosages for parenteral administration as described 
Table I Composition of tested peritoneal dialysis solutions

\begin{tabular}{|c|c|c|c|c|}
\hline \multirow[t]{2}{*}{ Components } & \multicolumn{2}{|c|}{$\begin{array}{l}\text { Low-GDP } \\
\text { glucose-based } \\
(w / v)\end{array}$} & \multicolumn{2}{|c|}{$\begin{array}{l}\text { Experimental } \\
(w / v)\end{array}$} \\
\hline & $1.36 \%^{\mathrm{a}}$ & $2.27 \%^{\mathrm{a}}$ & $0.70 \%$ & $1.50 \%$ \\
\hline Xylitol, mmol/L & - & - & 46 & 98.6 \\
\hline L-carnitine, $\mathrm{mmol} / \mathrm{L}$ & - & - & 1.24 & 1.24 \\
\hline Glucose, $\mathrm{mmol} / \mathrm{L}$ & 75.5 & 126 & 27.7 & 27.7 \\
\hline Sodium, mmol/L & 132 & 132 & 134 & 134 \\
\hline Calcium, mmol/L & 1.25 & 1.25 & 1.25 & 1.25 \\
\hline Chloride, mmol/L & 95 & 95 & 103 & 103 \\
\hline Magnesium, mmol/L & 0.25 & 0.25 & 0.25 & 0.25 \\
\hline Lactate, $\mathrm{mmol} / \mathrm{L}$ & 15 & 15 & 35 & 35 \\
\hline Bicarbonate, $\mathrm{mmol} / \mathrm{L}$ & 25 & 25 & - & - \\
\hline $\mathrm{pH}$ & 7 & 7 & 5.5 & 5.5 \\
\hline Osmolarity, mOsm/L $\mathrm{L}^{\mathrm{c}}$ & 344 & 395.5 & 349.5 & 402.3 \\
\hline
\end{tabular}

Notes: a ${ }^{6}$ lucose concentration (w/v). ${ }^{b}$ Xylitol concentration (w/v). ${ }^{c}$ Calculated. Abbreviation: GDP, glucose degradation product.

by the US Food and Drug Administration. ${ }^{32}$ Dulbecco's Modified Eagle's Medium (DMEM)-Low Glucose, M199 endothelial growth medium, penicillin-streptomycin, glutamine, phosphate-buffered saline, and $0.05 \%$ trypsin $/ 0.02 \%$ ethylenediaminetetraacetic acid (EDTA) were purchased from Mascia Brunelli (Milan, Italy). Fetal bovine serum was purchased from Gibco by Life Technologies (Monza, MB, Italy), and tissue-culture disposables were from Eppendorf (Hamburg, Germany). Phycoerythrin (PE)-labeled antiVascular Cell Adhesion Molecule-1 (anti-VCAM-1) and fluorescein isothiocyanate (FITC)-labeled anti-Intercellular Adhesion Molecule-1 (anti-ICAM-1) antibodies were from BioLegend (San Diego, CA, USA). Endothelial cell growth factor, bovine serum albumin, dimethyl sulfoxide, and 3-(4,5dimethylthiazolyl-2)-2,5-diphenyltetrazolium bromide (MTT) were from Sigma Aldrich (St Louis, MO, USA). HKGreen-4A probe was synthesized and kindly provided by Prof Dan Yang's lab. ${ }^{33}$

\section{Cell cultures and experimental procedures}

Umbilical cords were obtained from randomly selected healthy mothers (Control, C) and from mothers with GD delivering at the hospitals of Chieti and Pescara. All procedures were in agreement with the University G. d'Annunzio Chieti-Pescara Ethical Comittee (Reference Number: 1879/09COET) and with the Declaration of Helsinki principles. After obtaining approval of the protocol from the University G. d'Annunzio Chieti-Pescara Ethical Comittee, signed informed consent was obtained from each participating subject.

Primary HUVECs were obtained as described previously and used between the third and fifth passages in vitro. ${ }^{14}$ In this study, nine different HUVEC batches were employed and each experiment was performed on cells coming at least from three different batches. In all experiments, primary C- and GD-HUVECs were grown to confluency and exposed for 72 hours in 50:50 medium and PDSs (each of the four PDSs is reported in Table 1).

\section{Cell count}

After treatment with the four different PDSs (Table 1) for 72 hours, C- and GD-HUVECs were detached by using Trypsin-EDTA $\left(10 \mathrm{~min}\right.$ at $\left.37^{\circ} \mathrm{C}\right)$, resuspended in culture medium, and then counted with Burker's chamber.

\section{MTT assay}

The effect of the glucose-based and the experimental PDSs (Table 1) on HUVEC viability was assessed by the MTT method. Briefly, C- and GD-HUVECs were plated in 96-well tissue culture plates $\left(2,000 \mathrm{cells} / \mathrm{cm}^{2}\right)$ stimulated as described above and MTT solution $(0.5 \mathrm{mg} / \mathrm{mL})$ was added to each well and incubated for 3 hours. Then, $200 \mu \mathrm{L}$ of dimethyl sulfoxide was added to the cells for crystal solubilization. The spectrometric absorbance at $540 \mathrm{~nm}$ was read using a microplate reader (SpectraMAX 190; Molecular Devices, Sunnyvale, CA, USA).

\section{Intracellular peroxynitrite levels}

The intracellular levels of peroxynitrite $\left(\mathrm{ONOO}^{-}\right)$were detected in C- and GD-HUVECs stimulated with PDSs as described above by using the HKGreen-4A probe $(10 \mu \mathrm{M}$, $30 \mathrm{~min}$ at $37^{\circ} \mathrm{C}$ ), which was synthesized by Prof Dan Yang's lab. ${ }^{33}$

All data were analyzed using FACS Diva (BD Biosciences) and FlowJo ${ }^{\mathrm{TM}}$ Version 8.8.6 software (TreeStar, Ashland, OR, USA) and expressed as percentage of positive cells. ${ }^{34}$

\section{Adhesion molecules membrane exposure}

For fluorescence cytometry, C- and GD-HUVECs were stimulated as described in the experimental protocol and flow cytometry analysis performed as previously reported. ${ }^{35}$ Briefly, nonpermeabilized cells were detached by EDTA $5 \mathrm{mM}$ solution, washed, and resuspended in bovine serum albumin $(0.5 \%)$. Cells were pelleted by centrifugation at $800 \mathrm{rpm}$ for $15 \mathrm{~min}$ and then incubated with the primary antibodies anti-VCAM-1 PE-conjugate (1:100, PE; Biolegend) and anti-ICAM-1 FITC-conjugate (1:100, FITC), both for $30 \mathrm{~min}$ at room temperature. The incubation with primary antibody was followed by incubation with the specific 
FITC-labeled secondary antibody. All samples were analyzed on an FACS Canto II flow cytometer (BD Biosciences) using CellQuest ${ }^{\mathrm{TM}}$ software 3.2.1.f1 (BD Biosciences). ${ }^{36}$ Quality control included a regular check-up with Cytometer Setup and Tracking beads (BD Biosciences). Debris was excluded from the analysis by gating on morphological parameters; 10,000 nondebris events in the morphological gate were recorded for each sample. All antibodies were titrated under assay conditions and optimal photomultiplier gains were established for each channel. Data were analyzed using FlowJo Version 8.8.6 software (TreeStar) and expressed as mean fluorescence intensity (MFI) ratio. The MFI ratio was calculated by dividing the MFI of positive events by the MFI of negative events.

\section{Monocyte adhesion assays}

We evaluated U937 monocyte adhesion to C- and GDHUVECs using a cell adhesion assay in the normal growth condition (basal) and after incubation for 72 hours with the different PDSs (Table 1). Cells were grown to confluence in six-well tissue culture plates and U937 cell adhesion was evaluated as previously described. ${ }^{35}$ Briefly, $1 \times 10^{6}$ U937 cells/mL were added to each HUVEC monolayer under rotating conditions (63 rev/min) at room temperature. After $20 \mathrm{~min}$, nonadhering cells were removed and the monolayers were fixed with $1 \%$ paraformaldehyde. As experimental control, some monolayers were treated for 16 hours with tumor necrosis factor alpha $1 \mathrm{ng} / \mathrm{mL}$ and at 1 hour before the assay with mouse anti-human monoclonal antibody against VCAM-1 and ICAM-1. The number of adherent cells was assessed by counting eight different high-power fields $\left(3.5 \mathrm{~mm}^{2}\right)$. Photos of randomly chosen high-power fields were taken at half-radius distance from the center of the well in one of three comparative experiments of similar design, showing U937 monocytoid cell adhesion to C- and GD-HUVECs.

\section{Statistical analysis}

All experiments were repeated at least three times, and the results are presented as mean \pm standard deviation (SD). Statistical analysis was performed by the unpaired Student's $t$-test to compare basal C- and GD-HUVECs, or by one-way analysis of variance followed by the post hoc Bonferroni's multiple comparison test whenever the analysis of variance indicated the presence of a statistical significance, to compare the effects of the different PDSs. Significance was defined as a $P$-value $<0.05$.

\section{Results \\ Effect of PDSs on C- and GD-HUVECs viability}

Treatment with glucose-based PDSs (glucose 1.36\% and glucose $2.27 \%$ ) and with the two experimental PDSs (xylitol $0.7 \%$ and xylitol $1.5 \%$ ) for 72 hours did not alter the proliferative potential either in C- or in GD-HUVECs (data not shown). Notably, under the same experimental conditions, the experimental PDSs significantly improved C-HUVECs viability as compared to both glucose-based PDSs $(0.80 \pm 0.05$ and $0.88 \pm 0.03$ vs $0.54 \pm 0.06$ and $0.55 \pm 0.07$ $\mathrm{Abs}_{540 \mathrm{~nm}}, P<0.05$ for xylitol $0.7 \%$ and xylitol $1.5 \%$ vs glucose $1.36 \%$ and glucose $2.27 \%$, respectively) and basal condition $\left(0.80 \pm 0.05\right.$ and $0.88 \pm 0.03$ vs $0.51 \pm 0.05 \mathrm{Abs}_{540 \mathrm{~nm}}$, $P<0.05$ for xylitol $0.7 \%$ and xylitol $1.5 \%$ vs basal, respectively), while for GD-HUVECs, a positive trend that did not reach significance was found. In contrast, glucose-based PDSs did not improve cell viability both in C- and GDHUVECs (data not shown).

\section{Effect of PDSs on C- and GD-HUVECs nitro-oxidative stress}

In order to determine the potential protective effect of our experimental PDSs on nitro-oxidative stress, we evaluated the intracellular peroxynitrite levels in our cellular models.

Treatment with experimental PDSs significantly decreased peroxynitrite levels in GD-HUVECs, as compared to glucosebased PDSs and basal condition (Figure 1). In C-HUVEC

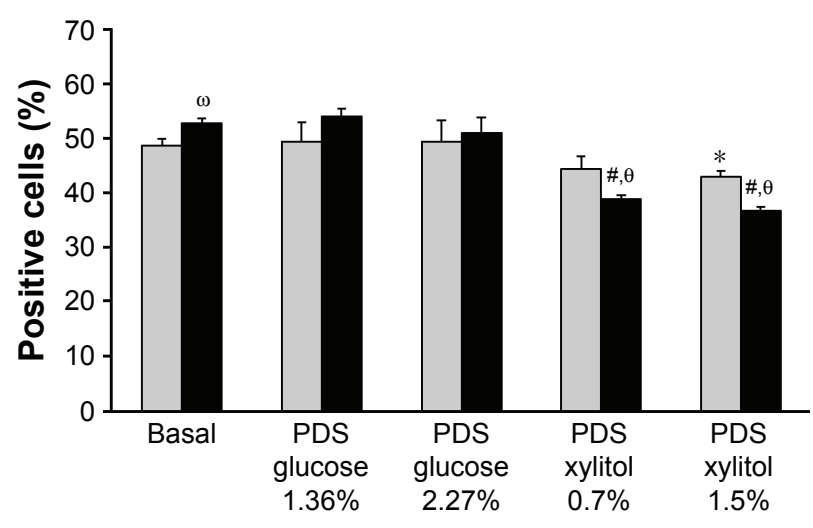

Figure I Effect of PDSs on intracellular peroxynitrite levels in C- and GD-HUVECs. Notes: Flow cytometry evaluation of peroxynitrite in C- (gray bars) and GDHUVECs (black bars) untreated (basal) or exposed to PDSs. Results are expressed as the percentage of peroxynitrite-positive cells $\pm S D$ of at least three different experiments. Student's $t$-test: ${ }^{\oplus} P<0.05$ versus $C$-HUVECs basal. Statistically significant difference in Bonferroni post hoc test: "versus GD-HUVECs basal and PDS glucose $1.36 \%$ and $2.27 \%,{ }^{\theta}$ versus C-HUVECs PDS xylitol $0.7 \%$ and $1.5 \%$, *versus C-HUVECs PDS glucose $1.36 \%$ and $2.27 \%$; for each symbol $P<0.05$.

Abbreviations: C, control; GD, gestational diabetes; HUVECs, Human Vein Endothelial Cells; PDS, peritoneal dialysis solution; SD, standard deviation. 
cultures, it was observed that xylitol-PDSs reduced nitrooxidative stress and this reached statistical significance at the concentration of $1.5 \%$ (Figure 1 ).

In addition, as previously demonstrated, unstimulated GD-HUVECs showed a significantly higher nitro-oxidative stress than C-HUVECs. ${ }^{14}$

\section{Effect of PDSs on adhesion molecule membrane exposure in C- and GD- HUVECs}

We next evaluated whether adhesion molecule membrane exposure might be modified upon treatment with the different PDSs.

As shown in Figure 2, following incubation with glucosebased PDSs, ICAM-1 (Figure 2A) and VCAM-1 (Figure 2B) membrane exposure increased in our cellular models, as compared to basal condition. Interestingly, our experimental PDSs decreased both ICAM-1 and VCAM-1 exposure compared to glucose-based PDSs, both in C- and GD-HUVECs (Figure 2). Furthermore, as previously demonstrated, at baseline, GD-HUVECs displayed higher ICAM-1 and VCAM-1 exposure levels than C-HUVECs. ${ }^{14}$

\section{Effect of PDSs on U937 monocyte adhesion to C- and GD-HUVECs}

By using the in vitro protocol of monocyte adhesion to the endothelium, which is close to the in vivo physiopathological state, we tested the effect of the four different PDSs on both C- and GD-HUVECs.

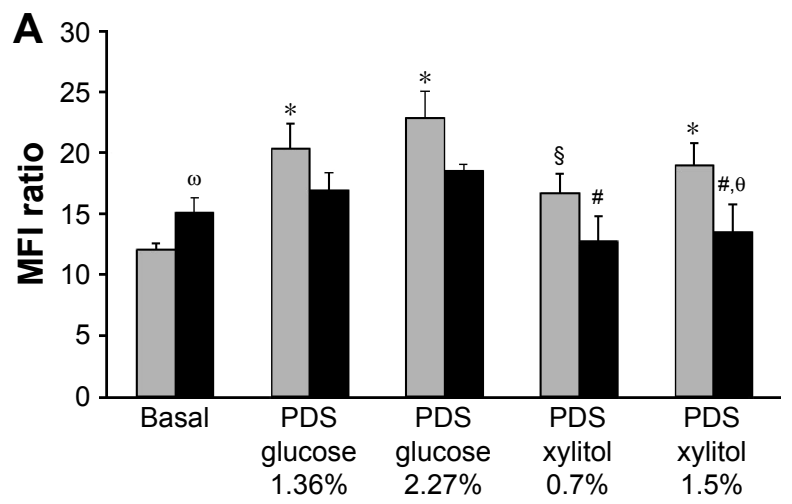

Stimulation with glucose-based PDSs caused an increase in the adhesion of monocytes to C- and GD-HUVECs, as compared to unstimulated cells (Figure 3 ). Of note, and as expected, this proinflammatory effect was absent when both C- and GD-HUVECs were exposed to our experimental PDSs, thus confirming the absence of proinflammatory vascular effects.

Interestingly, as compared to glucose-based PDSs, stimulation with xylitol-based PDSs induced a decreased trend in the adhesion of monocytes to GD-HUVECs, which reached statistical significance for C-HUVECs (Figure 3). Moreover, the hyperosmolar control (mannitol at doses of 30 and $60 \mathrm{mM}$ ) did not induce any effect on monocyte adhesion to C- and GD-HUVECs, indicating that the proinflammatory vascular effects of glucose-based PDSs were independent of their increased osmolarity. In addition, as previously demonstrated, at basal condition, monocyte adhesion to GD-HUVECs was significantly higher than that of C-HUVECs. ${ }^{14}$

Treating cells with anti-VCAM-1 or anti-ICAM-1 antibodies at saturating concentrations resulted in blocking U937 adhesion to both $\mathrm{C}$ - and GD-HUVECs, thus suggesting that hyperexpression of these molecules on the cell surface was among the main mechanisms for increased U937 adhesion to HUVECs (data not shown).

\section{Discussion}

In recent years, several studies have highlighted the detrimental effect of PDSs containing glucose on the longevity

Figure 2 Effect of PDSs on adhesion molecules exposure in C- and GD-HUVECs.

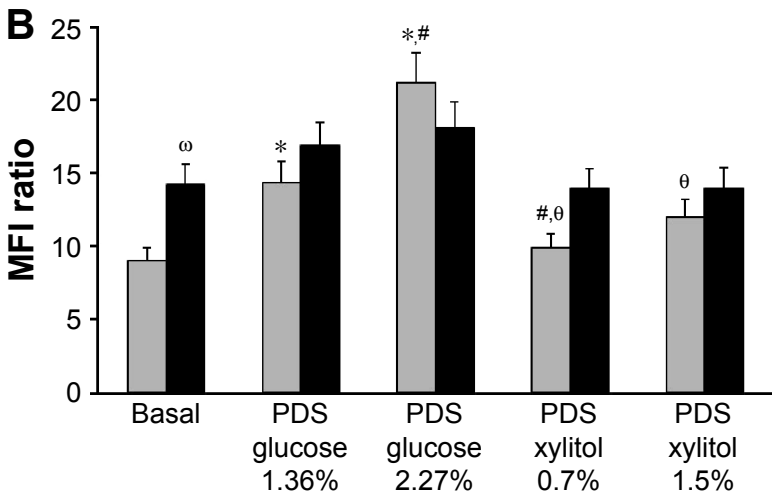

Notes: Flow cytometry evaluation of ICAM-I (A) and VCAM-I (B) membrane exposure in C- (gray bars) and GD-HUVECs (black bars) untreated (basal) or exposed to PDSs. ICAM-I and VCAM-I membrane exposure levels are expressed as fold increase \pm SD (at least three different experiments) of the MFI ratio versus basal condition. MFI ratio was calculated by dividing the MFI of positive events by the MFI of negative events. (A) Student's $t$-test: ${ }^{\omega} P<0.05$ versus $C$-HUVECs basal. Statistically significant difference in Bonferroni post hoc test: *versus C-HUVECs basal, ${ }^{\circledR}$ versus C-HUVECs PDS glucose $2.27 \%$, " versus GD-HUVECs PDS glucose $2.27 \%$, ${ }^{\theta}$ versus C-HUVECs PDS xylitol I.5\%; for each symbol $P<0.05$. (B) Student's $t$-test: ${ }^{\omega} P<0.02$ versus $C$-HUVECs basal. Statistically significant difference in Bonferroni post hoc test: *versus $C$-HUVECs basal, " versus C-HUVECs PDS glucose I.36\%, ${ }^{\theta}$ versusC-HUVECs PDS glucose $2.27 \%$; for each symbol $P<0.05$.

Abbreviations: C, control; GD, gestational diabetes; HUVECs, Human Vein Endothelial Cells; ICAM-I, Intercellular Adhesion Molecule-I; MFI, mean fluorescence intensity; PDS, peritoneal dialysis solution; SD, standard deviation; VCAM-I, Vascular Cell Adhesion Molecule-I. 
A

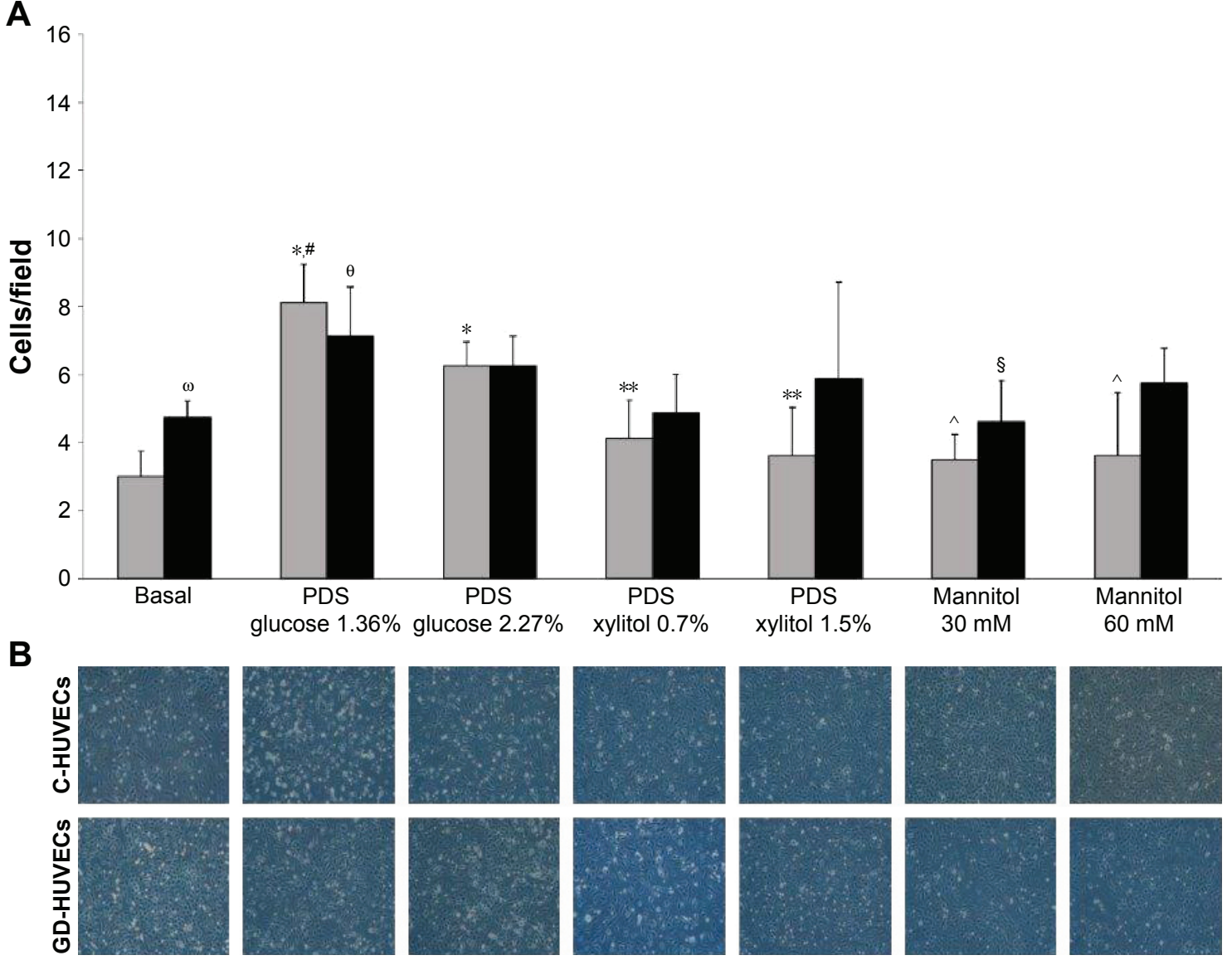

Figure 3 Effect of PDSs on monocyte adhesion to C- and GD-HUVECs.

Notes: (A) C- (gray bars) and GD-HUVECs (black bars) U937 interaction was evaluated in cells untreated (basal) or exposed to PDSs or to mannitol (as hyperosmolar control). Quantitative data show the number of $U 937$ cells adhering within a high-power field $\left(3.5 \mathrm{~mm}^{2}\right)$, with each measurement consisting of eight counts for every condition. Results are expressed as mean \pm SD of at least three different experiments. Student's $t$-test: ${ }^{\omega} P<0.05$ versus C-HUVECs basal. Statistically significant difference in Bonferroni post hoc test: *versus C-HUVECs basal, "versus C-HUVECs PDS glucose $2.27 \%$, ${ }^{\theta}$ versus GD-HUVECs basal, **versus C-HUVECs PDS glucose I.36\% and $2.27 \%$, ^versus C-HUVECs PDS glucose 1.36\%, ${ }^{\S}$ versus GD-HUVECs glucose 1.36\%; for each symbol P<0.05. (B) Representative images of U937 cell adhesion to C- and GD-HUVECs.

Abbreviations: C, control; GD, gestational diabetes; HUVECs, Human Vein Endothelial Cells; PDS, peritoneal dialysis solution; SD, standard deviation.

of PD patients. ${ }^{11,37,38}$ Hence, a major challenge of PD therapy is the development of glucose-sparing strategies that are able to provide an efficacious ultrafiltration profile without jeopardizing patient health.

Today, glucose sparing can be primarily offered by the use of PDSs containing the glucose polymer icodextrin or aminoacids as osmotic agents replacing glucose. These formulations, either alone or in combination, have been shown to be effective and PD patients may benefit from their use. ${ }^{18}$ However, both icodextrin and aminoacids can only replace $30 \%-50 \%$ of daily glucose absorption, ${ }^{11}$ and their use is limited to a single daily peritoneal exchange. ${ }^{39,40}$ Furthermore, two recent randomized, controlled studies (IMPENDIA and EDEN) showed that a low-glucose regimen based on dextrosebased solutes, icodextrin and aminoacids, though improving metabolic indices in diabetic PD patients, was associated with an enhanced risk of extracellular fluid volume expansion, causing an increase in serious adverse events and deaths. ${ }^{41}$
Thus, based on such findings, it is clear that the search for new solutions that manage to minimize the negative effects of $\mathrm{PD}$ represents an important objective. In the present study, we tested the biocompatibility of new experimental PDSs containing more than one osmo-metabolic agent, xylitol, glucose, and L-carnitine. Most of the osmotic strength of our PDSs is achieved by the presence of xylitol and carnitine, osmo-metabolic ingredients extremely stable from the chemical standpoint, even when steam-sterilized in an acidic environment (http://pubchem.ncbi.nlm.nih.gov).

In addition, the concept to introduce more than one osmo-metabolic agent in our PDSs is somehow derived from the well-known approach of polypharmacy or combination therapy, whereby the aim is to achieve a favorable synergetic action. ${ }^{42,43}$ Note that our experimental PDSs have a lower $\mathrm{pH}$ and a higher lactate concentration, conditions thought to affect biocompatibility of PD fluids, ${ }^{9}$ than the tested, commercially available, normal $\mathrm{pH}$, low-GDP PDS, which is regarded as 
a "biocompatible" solution. ${ }^{44}$ Our PDSs were also steamsterilized in a single-chambered bag containing a lactatebuffered glucose solution at $\mathrm{pH} 5.5$, a procedure known to generate more than fourfold acetaldehyde, a reliable indicator of GDPs, than in the two-chambered commercial bag tested in our study. ${ }^{45}$ The use of these xylitol-carnitine-based PDSs in our in vitro study proved not to change the proliferative potential in both C-and GD-HUVECs, compared to glucosebased PDSs. In addition, our PDSs significantly improved endothelial cell viability compared to basal condition.

Our results also show that glucose-based PDSs significantly increased VCAM-1 and ICAM-1 membrane exposure as compared to basal conditions in both C-and GD-HUVECs. Such proinflammatory vascular effect may have pathophysiologic consequences in the pathogenesis of atherosclerosis. In fact, increased expression of ICAM-1 and VCAM-1 on endothelial cell surface may promote adhesion of monocytes, which is a crucial event in vascular inflammation and the early atherosclerotic process. ${ }^{46}$ Moreover, upon being exposed on the endothelial cells, VCAM-1 and ICAM-1 can be released into the circulation; increased plasma levels of adhesion molecules, ${ }^{47}$ as found in PD patients, ${ }^{48}$ have been associated with cardiovascular events and RRF. ${ }^{49,50}$ Indeed, we found that glucose-based PDSs caused a significant increase in monocyte interaction with both $\mathrm{C}$ - and GD-HUVECs compared to basal condition. Notably, when endothelial cells were exposed to experimental PDSs, all the above unfavorable vascular effects were absent.

Thus, PD therapy seems to induce a significant proinflammatory effect on endothelial cells, which has been attributed to the high glucose concentrations and/or GDPs present in PD standard solutions. ${ }^{17}$ Although our experimental PDSs did contain some glucose (Table 1), this was not enough to trigger a comparable proinflammatory effect or nitro-oxidative stress as that seen for glucose-based PDSs in both C- and GD-HUVECs. This indicates that a small amount of glucose may be maintained in the PDS, in order to take advantage of its ultrafiltration ability and to provide energy source to patients who are often malnourished.

\section{Conclusion}

Our results show that in control and diabetic human endothelial cell models, xylitol-carnitine-based PDSs do not cause cytotoxicity and inflammation that are caused by the neutral pH, low-GDP hypertonic glucose-based PDSs. Since xylitol significantly inhibits hepatic glucose production, ${ }^{51}$ is a poor insulin secretagogue, and possesses a low glycemic index, whereas carnitine improves muscle glucose disposal, ${ }^{20}$ these findings suggest that osmo-metabolic-based PDSs may represent a desirable hypertonic solution even for diabetic patients in PD.

\section{Acknowledgments}

We gratefully acknowledge Daniela Heuberger for the excellent technical assistance. This work was in part supported by funds from Programma Operativo Nazionale (01_00937) - MIUR “Modelli sperimentali biotecnologici integrati per lo sviluppo e la selezione di molecole di interesse per la salute dell'uomo".

\section{Disclosure}

AA is an employee of CoreQuest. The authors report no other conflicts of interest in this work.

\section{References}

1. Jain AK, Blake P, Cordy P, Garg AX. Global trends in rates of peritoneal dialysis. J Am Soc Nephrol. 2012;23(3):533-544.

2. Mehrotra R, Chiu YW, Kalantar-Zadeh K, Bargman J, Vonesh E. Similar outcomes with hemodialysis and peritoneal dialysis in patients with endstage renal disease. Arch Intern Med. 2011;171(2):110-118.

3. Boateng EA, East L. The impact of dialysis modality on quality of life: a systematic review. J Ren Care. 2011;37(4):190-200.

4. Zhang AH, Cheng LT, Zhu N, Sun LH, Wang T. Comparison of quality of life and causes of hospitalization between hemodialysis and peritoneal dialysis patients in China. Health Qual Life Outcomes. 2007;5:49

5. Maiorca R, Brunori G, Zubani R, et al. Predictive value of dialysis adequacy and nutritional indices for mortality and morbidity in CAPD and HD patients. A longitudinal study. Nephrol Dial Transplant. 1995;10(12):2295-2305.

6. Bargman JM, Thorpe KE, Churchill DN; Group CPDS. Relative contribution of residual renal function and peritoneal clearance to adequacy of dialysis: a reanalysis of the CANUSA study. J Am Soc Nephrol. 2001; 12(10):2158-2162.

7. Termorshuizen F, Korevaar JC, Dekker FW, van Manen JG, Boeschoten EW, Krediet RT; NECOSAD Study Group. The relative importance of residual renal function compared with peritoneal clearance for patient survival and quality of life: an analysis of the Netherlands Cooperative Study on the Adequacy of Dialysis (NECOSAD)-2. Am J Kidney Dis. 2003;41(6):1293-1302.

8. Moist LM, Port FK, Orzol SM, et al. Predictors of loss of residual renal function among new dialysis patients. $J$ Am Soc Nephrol. 2000; 11(3):556-564.

9. Jorres A, Topley N, Gahl GM. Biocompatibility of peritoneal dialysis fluids. Int J Artif Organs. 1992;15(2):79-83.

10. Nilsson-Thorell CB, Muscalu N, Andren AH, Kjellstrand PT, Wieslander AP. Heat sterilization of fluids for peritoneal dialysis gives rise to aldehydes. Perit Dial Int. 1993;13(3):208-213.

11. Holmes CJ. Glucotoxicity in peritoneal dialysis - solutions for the solution! Adv Chronic Kidney Dis. 2007;14(3):269-278.

12. Diaz-Buxo JA, Gotloib L. Agents that modulate peritoneal membrane structure and function. Perit Dial Int. 2007;27(1):16-30.

13. Pandolfi A, De Filippis EA. Chronic hyperglicemia and nitric oxide bioavailability play a pivotal role in pro-atherogenic vascular modifications. Genes Nutr. 2007;2(2):195-208.

14. Di Fulvio P, Pandolfi A, Formoso G, et al. Features of endothelial dysfunction in umbilical cord vessels of women with gestational diabetes. Nutr Metab Cardiovasc Dis. 2014;24(12):1337-1345. 
15. Kim YL, Cho JH, Choi JY, Kim CD, Park SH. Systemic and local impact of glucose and glucose degradation products in peritoneal dialysis solution. J Ren Nutr. 2013;23(3):218-222.

16. Burkart J. Metabolic consequences of peritoneal dialysis. Semin Dial. 2004;17(6):498-504.

17. Caballo C, Palomo M, Cases A, et al. NFkappaB in the development of endothelial activation and damage in uremia: an in vitro approach. PLoS One. 2012;7(8):e43374.

18. Holmes C, Mujais S. Glucose sparing in peritoneal dialysis: implications and metrics. Kidney Int Suppl. 2006;(103):S104-S109.

19. Di Pietro N, Giardinelli A, Sirolli V, et al. Nitric oxide synthetic pathway and cGMP levels are altered in red blood cells from end-stage renal disease patients. Mol Cell Biochem. 2016;417(1-2):155-167.

20. Arduini A, Bonomini M, Savica V, Amato A, Zammit V. Carnitine in metabolic disease: potential for pharmacological intervention. Pharmacol Ther. 2008;120(2):149-156.

21. Bonomini M, Pandolfi A, Di Liberato L, et al. L-carnitine is an osmotic agent suitable for peritoneal dialysis. Kidney Int. 2011;80(6): 645-654.

22. Bonomini M, Di Liberato L, Del Rosso G, et al. Effect of an L-carnitinecontaining peritoneal dialysate on insulin sensitivity in patients treated with CAPD: a 4-month, prospective, multicenter randomized trial. Am J Kidney Dis. 2013;62(5):929-938.

23. Gaggiotti E, Arduini A, Bonomini M, et al. Prevention of peritoneal sclerosis: a new proposal to substitute glucose with carnitine dialysis solution (biocompatibility testing in vitro and in rabbits). Int $J$ Artif Organs. 2005;28(2):177-187.

24. Bazzato G, Coli U, Landini S, et al. Xylitol as osmotic agent in CAPD: an alternative to glucose for uremic diabetic patients? Trans Am Soc Artif Intern Organs. 1982;28:280-286.

25. Livesey G. Health potential of polyols as sugar replacers, with emphasis on low glycaemic properties. Nutr Res Rev. 2003;16(2):163-191.

26. Di Paolo N, Sacchi G. Atlas of peritoneal histology. Perit Dial Int. 2000;20(Suppl 3):S5-S96.

27. Thorgeirsson G, Robertson AL Jr. The vascular endothelium-pathobiologic significance. Am J Pathol. 1978;93(3):803-848.

28. Ozuguz U, Isik S, Berker D, et al. Gestational diabetes and subclinical inflammation: evaluation of first year postpartum outcomes. Diabetes Res Clin Pract. 2011;94(3):426-433.

29. Wang AY. Consequences of chronic inflammation in peritoneal dialysis. Semin Nephrol. 2011;31(2):159-171.

30. Stenvinkel P, Ketteler M, Johnson RJ, et al. IL-10, IL-6, and TNF-alpha: central factors in the altered cytokine network of uremia - the good, the bad, and the ugly. Kidney Int. 2005;67(4):1216-1233.

31. Locatelli F, Canaud B, Eckardt KU, Stenvinkel P, Wanner C, Zoccali C. Oxidative stress in end-stage renal disease: an emerging threat to patient outcome. Nephrol Dial Transplant. 2003;18(7):1272-1280.

32. Schneider AS, Schettler A, Markowski A, et al. Assessment of xylitol serum levels during the course of parenteral nutrition including xylitol in intensive care patients: a case control study. Clin Nutr. 2014;33(3): 483-488.

33. Peng T, Wong NK, Chen X, et al. Molecular imaging of peroxynitrite with HKGreen-4 in live cells and tissues. J Am Chem Soc. 2014;136(33): $11728-11734$
34. Lanuti P, Rotta G, Almici C, et al. Endothelial progenitor cells, defined by the simultaneous surface expression of VEGFR2 and CD133, are not detectable in healthy peripheral and cord blood. Cytometry A. 2016;89(3):259-270.

35. Di Tomo P, Di Silvestre S, Cordone VG, et al. Centella asiatica and lipoic acid, or a combination thereof, inhibit monocyte adhesion to endothelial cells from umbilical cords of gestational diabetic women. Nutr Metab Cardiovasc Dis. 2015;25(7):659-666.

36. Lanuti $\mathrm{P}$, Marchisio M, Cantilena S, et al. A flow cytometry procedure for simultaneous characterization of cell DNA content and expression of intracellular protein kinase C-zeta. J Immunol Methods. 2006;315(1-2):37-48.

37. Davies SJ, Phillips L, Griffiths AM, Russell LH, Naish PF, Russell GI. What really happens to people on long-term peritoneal dialysis? Kidney Int. 1998;54(6):2207-2217.

38. Chaudhary K, Khanna R. Biocompatible peritoneal dialysis solutions: do we have one? Clin J Am Soc Nephrol. 2010;5(4):723-732.

39. Jones M, Hagen T, Boyle CA, et al. Treatment of malnutrition with $1.1 \%$ amino acid peritoneal dialysis solution: results of a multicenter outpatient study. Am J Kidney Dis. 1998;32(5):761-769.

40. Johnson DW, Agar J, Collins J, et al. Recommendations for the use of icodextrin in peritoneal dialysis patients. Nephrology (Carlton). 2003; 8(1):1-7.

41. Li PK, Culleton BF, Ariza A, et al. Randomized, controlled trial of glucose-sparing peritoneal dialysis in diabetic patients. $J$ Am Soc Nephrol. 2013;24(11):1889-1900.

42. American Diabetes Association. (7) Approaches to glycemic treatment. Diabetes Care. 2015;38(Suppl 1):S41-S48.

43. Taddei S. Combination therapy in hypertension: what are the best options according to clinical pharmacology principles and controlled clinical trial evidence? Am J Cardiovasc Drugs. 2015;15(3):185-194.

44. Seo EY, An SH, Cho JH, et al. Effect of biocompatible peritoneal dialysis solution on residual renal function: a systematic review of randomized controlled trials. Perit Dial Int. 2014;34(7):724-731.

45. Cooker LA, Luneburg P, Faict D, Choo C, Holmes CJ. Reduced glucose degradation products in bicarbonate/lactate-buffered peritoneal dialysis solutions produced in two-chambered bags. Perit Dial Int. 1997;17(4):373-378.

46. Ross R. Atherosclerosis - an inflammatory disease. $N$ Engl J Med. 1999;340(2):115-126.

47. Gearing AJ, Newman W. Circulating adhesion molecules in disease. Immunol Today. 1993;14(10):506-512.

48. Bonomini M, Reale M, Santarelli P, Stuard S, Settefrati N, Albertazzi A. Serum levels of soluble adhesion molecules in chronic renal failure and dialysis patients. Nephron. 1998;79(4):399-407.

49. Wang AY, Lam CW, Wang M, et al. Circulating soluble vascular cell adhesion molecule 1: relationships with residual renal function, cardiac hypertrophy, and outcome of peritoneal dialysis patients. Am J Kidney Dis. 2005;45(4):715-729.

50. Choi HY, Lee JE, Han SH, et al. Association of inflammation and protein-energy wasting with endothelial dysfunction in peritoneal dialysis patients. Nephrol Dial Transplant. 2010;25(4):1266-1271.

51. Kishore P, Kehlenbrink S, Hu M, et al. Xylitol prevents NEFA-induced insulin resistance in rats. Diabetologia. 2012;55(6):1808-1812.
Drug Design, Development and Therapy

\section{Publish your work in this journal}

Drug Design, Development and Therapy is an international, peerreviewed open-access journal that spans the spectrum of drug design and development through to clinical applications. Clinical outcomes, patient safety, and programs for the development and effective, safe, and sustained use of medicines are the features of the journal, which

\section{Dovepress}

has also been accepted for indexing on PubMed Central. The manuscript management system is completely online and includes a very quick and fair peer-review system, which is all easy to use. Visit http://www.dovepress.com/testimonials.php to read real quotes from published authors. 